Brit. F. industr. Med. 1967, 24, 71

\title{
Determination of the Carboxyhaemoglobin Saturation of Blood by Spectrophotometric Analysis
}

\author{
J. M. BEECKMANS \\ From the Faculty of Engineering Science, University of Western Ontario, London, Ontario, Canada
}

The spectrophotometric method of Commins and Lawther (1965) for the determination of carboxyhaemoglobin saturation in blood was found to lead to systematic undervaluation, because of dissociation of the carboxyhaemoglobin, and physical dissolution of the carbon monoxide in the solvent used for the analysis. The theoretical relationship between the true and apparent carboxyhaemoglobin saturations was calculated and was found to be in satisfactory agreement with the experimental findings, using samples of blood saturated with carbon monoxide, and aerated solvent. The error is much smaller when using oxygenfree solvent, but it is nevertheless appreciable at high carboxyhaemoglobin saturations.

An elegant method for the determination of carboxyhaemoglobin saturation, using a very small sample of blood, was recently described in this journal by Commins and Lawther (1965). The method requires $0.01 \mathrm{ml}$. of blood, which is dissolved in 10 $\mathrm{ml}$. of $0.04 \%$ ammonia solution. Measurements of the optical density differences at $4 \mathrm{I} 4 \mathrm{~m} \mu, 420.2 \mathrm{~m} \mu$, and $426 \mathrm{~m} \mu$ between this solution and a portion thereof from which all carboxyhaemoglobin has been converted to oxyhaemoglobin by bubbling oxygen give an estimate of the carboxyhaemoglobin present in the original sample. The method is calibrated by using a part of the same solution which has been saturated with carbon monoxide.

The purpose of this note is to point out that a systematic error occurs, because of dissociation of the carboxyhaemoglobin caused by physical dissolution of the carbon monoxide in the solution. This error can be serious, particularly at high carboxyhaemoglobin concentration. Commins and Lawther stated that preliminary experiments showed that the analysis gave the same result whether or not the solution was oxygen-free. This has not been the experience of the writer, who found an error which was in good agreement with prediction for airsaturated solutions.

Consider a blood sample of volume $V_{b}$, with oxygen or carbon monoxide binding capacity of $r$ ml. S.T.P.D. per ml. of blood. Let the carboxyhaemoglobin saturation be $S_{0}$. Then the total quantity of carbon monoxide in this sample is:

Received for publication May 17, 1966.

$$
Q_{0}=V_{b} r S_{0} \quad \text { ml. S.T.P.D. }
$$

This blood sample is now dissolved in ammonia solution to a volume $V_{s}$. Let the new carboxyhaemoglobin saturation be $S$, and let ' $a$ ' be the Henry's law constant for carbon monoxide in the solution. Thus ' $a$ ' equals the volume, in ml., of carbon monoxide, at S.T.P.D., dissolved per $\mathrm{ml}$. of solution, at I atmosphere pressure. The actual pressure of carbon monoxide in the solution is:

$$
\mathrm{p}_{\mathrm{co}}=\frac{P}{M} \frac{S}{\mathrm{I}-S}
$$

where $P$ is the oxygen partial pressure, in atmospheres, and $M$ is Haldane's constant. Then

$$
Q_{0}=V_{b} r S_{0}=V_{b} r S+\operatorname{ap}_{c o} V_{s}
$$

or, substituting for $\mathrm{p}_{\text {co }}$ from equation 2 ,

$$
V_{b} r S_{\mathrm{o}}=V_{b} r S+\frac{\mathrm{aP} V_{s}}{M} \frac{S}{\mathrm{I}-S}
$$

Hence

$$
\begin{gathered}
S_{\mathrm{o}}=S+\frac{\mathrm{aP} V_{s}}{M r V_{b}} \frac{S}{\mathrm{I}-S} \\
S_{0}=S\left(\mathrm{I}+\frac{K}{\mathrm{I}-S}\right)
\end{gathered}
$$

where

$$
K=\mathrm{aP} V_{s} / M r V_{b}
$$

Thus the observed carboxyhaemoglobin saturation must be multiplied by a factor $I+K /(I-S)$ to give the original value. The factor $K$ equals the fractional error at the limit of zero carboxyhaemoglobin content. The observed fractional error thus always exceeds $K$. For a $V_{s} / V_{b}$ ratio of $10^{3}$, as used by Commins and Lawther, and using values 
of 240 for $M, 0.21$ for $\mathrm{P}, 0.2$ for $r$, and 0.0214 for ' $a$ ' (taken from the Handbook of Chemistry and Physics, for carbon monoxide in water at $25^{\circ} \mathrm{C}$.), the value of $K$ is found to be 0.094 .

The calculated value of $K$ was confirmed experimentally by determining the apparent carboxyhaemoglobin content of samples of saturated human blood. A sample of blood was drawn with a syringe and diluted with one-tenth of its volume of $0.1 \mathrm{M}$ sodium oxalate anticoagulant. Pure carbon monoxide was drawn into the syringe and allowed to come into equilibrium with the blood. After equilibration the carbon monoxide gas was expelled, and the residual gas in the syringe was mixed with methane. This was done to ensure that the error introduced by physical dissolution of carbon monoxide in the plasma was negligible. The apparent carboxyhaemoglobin content of the blood was then determined by the method of Commins and Lawther, using a Beckman D.U. spectrophotometer with the slit set at $0.2 \mathrm{~mm}$. Equation 6 may be solved for $K$ if $S_{0}$ is known. For $S_{0}$ equal to unity, we have:

$$
K=\frac{(1-S)^{2}}{S}
$$

Six values of $S$ were determined for each of two ratios $V_{s} / V_{b}$, for both aerated and the oxygen-free solutions, and the results are shown in Table I. The

\section{TABLE I}

Apparent Carboxyhaemoglobin Saturations for Aerated AND OXYGEN-FREE SOLUTIONS

\begin{tabular}{|c|c|c|c|c|c|}
\hline & & \multicolumn{2}{|c|}{$\begin{array}{l}\text { Aerated } \\
\text { Solutions }\end{array}$} & \multicolumn{2}{|c|}{$\begin{array}{l}\text { Oxygen-free } \\
\text { Solutions }\end{array}$} \\
\hline & & $A$ & $B$ & $C$ & $D$ \\
\hline \multirow[t]{2}{*}{$V_{s} / V_{b} \times 10^{-3}$} & . & 0.625 & $1 \cdot 25$ & 0.625 & $1 \cdot 25$ \\
\hline & & $\begin{array}{l}0.793 \\
0.752 \\
0.793 \\
0.785 \\
0.780 \\
0.765\end{array}$ & $\begin{array}{l}0.697 \\
0.660 \\
0.712 \\
0.663 \\
0.708 \\
0.680\end{array}$ & $\begin{array}{l}0.870 \\
0.917 \\
0.873 \\
0.907 \\
0.910 \\
0.903\end{array}$ & $\begin{array}{l}0.878 \\
0.858 \\
0.892 \\
0.833 \\
0.879 \\
0.830\end{array}$ \\
\hline Mean & . & 0.778 & 0.687 & 0.897 & 0.862 \\
\hline
\end{tabular}

$K$ corresponding to the mean $S$ for each set of results, and the calculated standard $K$ s, referred to a $V_{s} / V_{b}$ ratio of $10^{3}$, are given in Table II. It is seen that the experimental values of $K^{\circ}$ are in satisfactory agreement with the computed value 0.094 .

It should be noted that the foregoing theoretical analysis does not apply in the case of oxygen-free
TABLE II

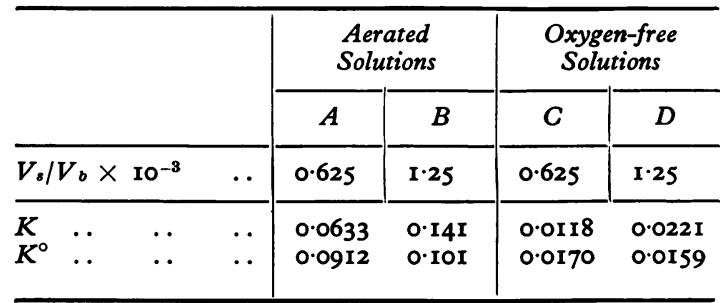

$K^{\circ}$ equals $0.9 \times 10^{-3} \mathrm{KV}_{b} / V_{8}$. The factor 0.9 arises from the dilution of the blood with anticoagulant.

solutions, since in this case equation 2 predicts a zero partial pressure for carbon monoxide, and hence $K^{\circ}$ of zero. Furthermore, equation 2 is not applicable to the equilibrium between carbon monoxide, carboxyhaemoglobin, and reduced haemoglobin, which is the appropriate equilibrium reaction in oxygen-free solutions. Since oxygen competes for haemoglobin with carbon monoxide, the equilibrium partial pressure of carbon monoxide at a given carboxyhaemoglobin concentration is always lowered when the partial pressure of oxygen is lowered, even in the presence of reduced haemoglobin. The effect of reducing the oxygen pressure on the system under consideration is thus to reduce the observed $K$, if other conditions are held constant. The lowest observed $K$ will be obtained with oxygen-free solutions: it will have a positive value because dissociation of the carboxyhaemoglobin will still take place. There is, however, no reason to believe that in this case $K^{\circ}$ will not be a function of $V_{b} / V_{s}$ or of $S_{0}$. The exact relationship between $K^{\circ}$ and these variables will in theory be a function of the equilibrium curve between carbon monoxide, reduced haemoglobin, and carboxyhaemoglobin. It has not been worked out, because in practice some residual oxygen will usually be present, and in any case, for moderate carboxyhaemoglobin concentrations, the error correction is so small in low or zero oxygen solutions that it will probably be adequate in most applications to assume a constant value of $K^{\circ}$, irrespective of $S_{0}$, and to assume that $K$ equals $K^{\circ} V_{s} / V_{b} \times 10^{-3}$. At high values of $S_{0}$, it is probably preferable to work with solutions of known oxygen tension, since the correction will be known with greater certainty. Under all conditions, the correction may be reduced by working with a higher blood concentration, which may necessitate using an absorbance cell with a short light path.

\section{REFERENCE}

Commins, B. T., and Lawther, P. J. (1965). Brit. F. industr. Med., 22, 139. 